\title{
Leaning on Community-Based Participatory Research to Respond During COVID-19
}

\author{
Annie L. Nguyen ${ }^{1} \cdot$ Christopher Christensen ${ }^{2} \cdot$ Jeff Taylor $^{2} \cdot$ Brandon Brown $^{3}$
}

Published online: 14 May 2020

(c) Springer Science+Business Media, LLC, part of Springer Nature 2020

\section{Introduction}

Prior to COVID-19, the HIV epidemic was arguably the worst public health crisis affecting the United States since the 1918 flu pandemic. While previously a death sentence, combination therapy transformed HIV infection into a largely manageable, chronic condition, leading to the majority of people with HIV being over age 50 [1]. We have little understanding of the physical and psychosocial needs of this first cohort to age with HIV. Challenges associated with aging with HIV are compounded by discrimination due to race, gender, and sexual orientation. Many people aging with HIV have also endured significant trauma due to AIDS, including personal losses of friends and loved ones, contributing to high rates of depression [2].

In mid-March 2020, the COVID-19 pandemic prompted "shelter-in-place" orders around the country. Older adults living with HIV are at increased risk for severe COVID19 complications due several factors including accentuated aging, being immunocompromised, and having higher rates of serious co-morbid conditions such as diabetes, cardiovascular and kidney diseases [3, 4]. People with complicating health factors are cautioned by the Centers for Disease Control and Prevention to take extra precautions to avoid COVID-19. So while physical distancing is a necessary strategy to flatten the curve, ease pressure on the healthcare system, and protect the most vulnerable, the order further increases the isolation faced by people aging with HIV, and

Annie L. Nguyen

annie.nguyen@med.usc.edu

1 Department of Family Medicine, Keck School of Medicine, University of Southern California, Los Angeles, CA, USA

2 HIV + Aging Research Project- Palm Springs (HARP-PS), Palm Springs, CA, USA

3 Department of Social Medicine, Population and Public Health, University of California, Riverside School of Medicine, Riverside, CA, USA creates barriers to accessing medications, health services, and other resources. For many long-term HIV survivors, the current COVID-19 pandemic parallels the early days of AIDS in many ways, with mirrored delays in acknowledging and responding to the pandemic and lack of testing and treatment.

\section{Capacity to Respond to Needs and Community-Based Participatory Research}

We partnered with the HIV + Aging Research Project, a community-based, nonprofit organization founded as a collaboration between local HIV clinicians and people living with HIV, to study the clinical and psychosocial aspects of aging among long-term HIV survivors. We obtained a Patient-Centered Outcomes Research Institute (PCORI) engagement award, which was meant to build local capacity to conduct research through community-based participatory research (CBPR) approaches. Located in Palm Springs, California (a popular retirement community in the Coachella Valley with the highest proportion of people aging with HIV in the United States), we have been working over the past five years to prepare stakeholders to conduct innovative and local research. As part of this, we formed a steering committee led by research and community member co-leaders, and including older adults living with HIV, non-medical caregivers of older adults with HIV, community-based organizations that serve people living with HIV, researchers, and healthcare providers. The steering committee meets on a monthly basis, with a core team meeting weekly to be able to rapidly respond to emerging health concerns and priorities. As the COVID-19 pandemic arrived, and the committee shifted to remote meetings, the urgency of the pandemic and understanding the unmet needs of the community aging with HIV became apparent. Since the committee is responsive to current needs, they shifted their efforts to COVID-19 with the 
idea to conduct a needs and post-traumatic stress disorder (PTSD) assessment survey of older adults living with HIV in the Coachella Valley. All stakeholders played a role in developing question topics and helped guide the creation of the survey to be most beneficial to people aging with HIV.

\section{A Rapid Response Needs Assessment}

Recognizing the need for a rapid response, we designed the needs assessment as a one-time, anonymous, internet-based survey to assess the impact of COVID-19 and mitigation efforts (i.e., shelter-in-place mandates, physical distancing guidelines) on daily needs, mental and physical health needs, and social isolation. We utilized tools from the National Institutes of Health Disaster Research Response database (www.dr2.nlm.nih.gov), and instruments from researchers engaged in the field of HIV and aging. The initial survey was presented virtually at a steering committee meeting, and extensive feedback was received from community members and other stakeholders. Once we reached consensus on questions, we then piloted the survey with 10 participants to identify steps in the outreach protocol or instruments that needed refinement. In the implementation phase of the study, we reached out to local service-based groups in the Coachella Valley known to assist older adults living with HIV and distributed recruitment emails to their members through their mailing lists. We were able to move from idea germination to survey implementation in less than three weeks.

Data collection are ongoing but we report some initial, descriptive findings here $(\mathrm{N}=35)$. Respondents were predominantly white $(91.4 \%)$, gay men (91.4\%) with a mean age of 63.5 years $(\mathrm{SD}=7.6)$ and a mean of 26.1 years $(\mathrm{SD}=8.6)$ living with HIV. Most participants (62.9\%) reported that COVID-19 impacted their lives "extremely" or "very much" and $60.0 \%$ were fearful of getting COVID-19 themselves, while $82.9 \%$ were worried about friends, family, and loved ones getting COVID-19. Some participants (20.0\%) stated that they were confused about where to obtain true and accurate information about COVID-19.

Frustration (48.6\%), boredom (42.9\%), sleep disruptions (48.6\%), and not getting enough exercise $(65.7 \%)$ were commonly reported by participants. Half (54.3\%) reported experiencing anxiety and $22.9 \%$ reported feelings of depression. In terms of substance use behaviors, $20.0 \%$ reported increases in alcohol use, $14.3 \%$ reported increases in marijuana use, and 5.7\% reported increases in other substance use during the pandemic. One-third experienced financial challenges because of COVID-19, 20.0\% experienced difficulty paying for basic needs like food, electricity, and rent, and $34.3 \%$ state that they or someone in their household have requested unemployment benefits. However, only $11.4 \%$ have received unemployment benefits.
Some participants (11.4\%) reported skipping meals or reducing portions because they were worried they didn't have enough money for food.

Over a quarter of participants $(28.6 \%)$ reported that they missed a dose of their HIV medication during the COVID-19 pandemic, with the majority stating that they forgot as the reason for missing a dose. Several people further explained that COVID-19 has been "a disorienting event" and that being "more stressed = more forgetful." On a Likert scale ranging from 1 (not at all) to 10 (extremely), participants reported a mean of $6.3(\mathrm{SD}=2.6)$ for feeling socially isolated, with $41.3 \%$ reporting in the upper range of 8 to 10 .

\section{Implications for Community Providers}

Our initial findings suggest COVID-19 has significant impacts on mental health and exercise behaviors as well as negative implications for financial well-being. Importantly for people aging with HIV, COVID-19 appears to be causing disruption to medication adherence. In addition to the initial findings presented here, we reiterate the key role of CBPR in making this study possible. The speed at which we were able to respond was due to several factors: (1) our team's existing networks of experts in HIV and aging, (2) an established steering committee with members who are familiar with reviewing and providing feedback about research, (3) relationships that have cultivated trust between academics and community members over time, and (4) a responsive institutional review board.

Our next step is to provide data in real-time to local service based groups in the Coachella Valley, so organizations can respond to the emergent needs of older adults living with HIV. At the completion of the study, findings will contribute to understanding the impact of COVID19 on older adults living with HIV with implications for emergency preparedness in order to better prepare for the ongoing threat of COVID-19 and the next public health crisis or pandemic. There will not be an immediate end to the COVID-19 pandemic and people in the most vulnerable groups, including people aging with HIV, may need to shelter-in-place long after much of their surrounding community is able to return to a semblance of normal life.

Acknowledgements We would like to thank the members of the HARP-PS steering committee for their valuable input, and our PLWH community for their continuing altruism and support of this research. We also acknowledge funding from the USC Clinical and Translational Science Institute (1UL1TR001855), the Patient Centered Outcomes Research Institute (4367-RUOC), and NIH/NIA (K01 AG064986-01) to A.N. These funding agencies had no role in study design, data collection and analysis, decision to publish, or preparation of the manuscript. 


\section{References}

1. HIV and Older Americans. Centers for Disease Control and Prevention website. https://www.cdc.gov/hiv/group/age/olderameri cans/index.html. Updated November 12, 2019. Accessed May 4, 2020.

2. Halkitis PN, Krause KD, Vieira DL. Mental health, psychosocial challenges and resilience in older adults living with HIV. Interdiscip Top Gerontol Geriatr. 2017;42:187-203. https://doi. org/10.1159/000448564.

3. Cahill S, Valadez R. Growing older with HIV/AIDS: new public health challenges. Am J Public Health. 2013;103(3):e7-e15. https ://doi.org/10.2105/AJPH.2012.301161.
4. Marg LZ, Heidari O, Taylor J, Marbley C, Scheibel S, Hagan R, Messaoudi I, Mendoza N, Brown B. A multidimensional assessment of successful aging among older people living with HIV in Palm Springs, California. AIDS Res Hum Retroviruses. 2019;35(11-12):1174-80. https://doi.org/10.1089/ AID.2019.0048.

Publisher's Note Springer Nature remains neutral with regard to jurisdictional claims in published maps and institutional affiliations. 